\title{
bioengineering
}

ISSN 2306-5354

www.mdpi.com/journal/bioengineering

Communication

\section{Biocatalytic Synthesis of Fluorescent Conjugated Indole Oligomers}

\section{Ryan M. Bouldin ${ }^{1}$, Ankita Singh ${ }^{2,3}$, Michael Magaletta ${ }^{2}$, Sean Connor ${ }^{2}$, Jayant Kumar ${ }^{3,4}$ and} Ramaswamy Nagarajan $2,3, *$

1 Department of Natural and Applied Sciences, Bentley University, Waltham, MA 02452, USA;

E-Mail: rbouldin@bentley.edu

2 Department of Plastics Engineering, University of Massachusetts, Lowell, MA 01854, USA;

E-Mails: ankita_singh@student.uml.edu (A.S.); michael_magaletta@student.uml.edu (M.M.); sean_connor@student.uml.edu (S.C.)

3 Center for Advanced Materials, University of Massachusetts, Lowell, MA 01854, USA;

E-Mail: jayant_kumar@uml.edu

4 Department of Physics, University of Massachusetts, Lowell, MA 01854, USA

* Author to whom correspondence should be addressed; E-Mail: Ramaswamy_Nagarajan@uml.edu; Tel.: +1-978-934-3454.

External Editor: Anthony Guiseppi-Elie

Received: 28 July 2014; in revised form: 18 September 2014 / Accepted: 27 November 2014 /

Published: 3 December 2014

\begin{abstract}
Fluorescent conjugated materials exhibiting reasonable biocompatibility that are capable of interacting with biological molecules are of interest for bio-sensing and imaging applications. Traditional approaches do not allow for the synthesis of conjugated materials in the presence of biologically relevant substrates. Further conjugated polymers synthesized using conventional methods are doped and not fluorescent. Here we explore the possibility of synthesizing fluorescent oligomers of indole using enzymes as catalyst under mild conditions. The peroxidase catalyzed coupling reaction presented here creates a photoluminescent material that allows for direct utilization (without purification and separation of the dopant) in biosensing applications. The polymerization reaction proceeds smoothly in just deionized water and ethanol. Monitoring of the absorption and fluorescence spectra over one hour shows that the concentration of both absorbing and emitting species grows steadily over time. The presence of anionic buffers and templates is shown to effectively retard the development of light emitting species and instead leads to the formation of an electrically doped conjugated polymer. Structural characterization
\end{abstract}


through FTIR and ${ }^{1} \mathrm{H}-\mathrm{NMR}$ analysis suggests that the oligomer is coupled through the 2 and 3 positions on the indole ring.

Keywords: polyindole; indole oligomer; biocatalyic; oxidoreductase; enzymatic polymerization; soybean peroxidase

\section{Introduction}

Conjugated polymers (CP) have attracted considerable attention from materials scientists, chemists and biologists, due to their tunable optical and electrical properties, rendering them suitable for applications in organic electronics and biosensing [1]. Optical sensors based on fluorescence quenching or 'turn-on' of luminescence have been most popular in biosensing applications [2]. The detection schemes for these sensors employ the light harvesting or conformation sensitive optical properties of the CP. The presence/binding of biomolecules or other relevant biological events is detected through Förster resonance energy transfer (FRET) between the $\mathrm{CP}$ and an acceptor molecule, or these biomolecules affect the conformation of the conjugated backbone leading to a detectable change in optical properties of the CP [3]. $\mathrm{CP}$ have evolved to become novel molecular tools for detection and imaging of biological processes in a complementary fashion to conventional techniques [4].

Key considerations in the design of fluorophores have often included high quantum yield of emission, solubility, ability to interact with biomolecules and a reasonable level of biocompatibility (allowing for detection or imaging without denaturing the biological entity). Solubility of fluorophores (such as conjugated polymers) in predominantly aqueous media is essential for interfacing of the fluorophore with biomacromolecules, such as proteins or nucleic acids [5]. It is becomingly increasingly clear that even for fluorescence-based in vivo imaging applications water solubility and reasonable biocompatibility of the fluorophores are crucial [6].

However, most conjugated polymers are intractable and not inherently water-soluble. Incorporation of pendant water-soluble moieties on conjugated polymers is one of the common strategies adopted to improve water solubility. Water-soluble moieties allow for tuning the interactions of conjugated polymers with biomolecules. Super-quenching of water-soluble poly(phenylene vinylene)s and poly(phenylene ethynylene)s has been reported in fluorescence turn-on and turn-off protein assays [7]. In these cases, the energy transfer between poly(fluorene-co-phenylene) derivatives and an acceptor dye is often utilized for fluorescence turn-on protein assays with amplified signal output.

For improved interaction between $\mathrm{CP}$ and biological polyelectrolytes such as DNA, cationic or anionic groups have been incorporated in the side chain of $\mathrm{CP}$ such as polythiophene. Cationic polythiophenes have been used for colorimetric detection of oligonucleotide hybridization based on conformational changes of the polymer upon interaction with DNA [3]. Signal transduction is typically controlled by electrostatic interactions between the charged species on the side chain of CP and the biological entities.

There is obviously a need for new non-toxic fluorescent conjugated materials that can be synthesized in predominantly aqueous media and near neutral $\mathrm{pH}$ conditions. The possibility of synthesizing $\mathrm{CP}$ under these conditions can also provide the opportunity to improve the interaction of the $\mathrm{CP}$ with biomolecules. In order to explore this aspect, the polymerization of indole monomers is investigated in 
this paper. Indole monomers and its derivatives are known to be fluorescent in their native state [8]. The fluorescence of indole has been utilized in the quantification of indole-conjugated proteins. Indoles have also been chemically or electrochemically polymerized to yield doped polyindoles with semi-conducting properties. The polymerization of indole has been shown to proceed through different routes yielding at least three distinct types of non-planar polymers [9,10]. Polyindoles have been reported to be reasonably stable, with good redox activity and electrical conductivity close to $0.1 \mathrm{~S} / \mathrm{cm}$ in the doped state [11]. Figure 1 represents the different possible coupling sites and chemical structures of polyindole.

Figure 1. Possible coupling sites for the polymerization of indole.

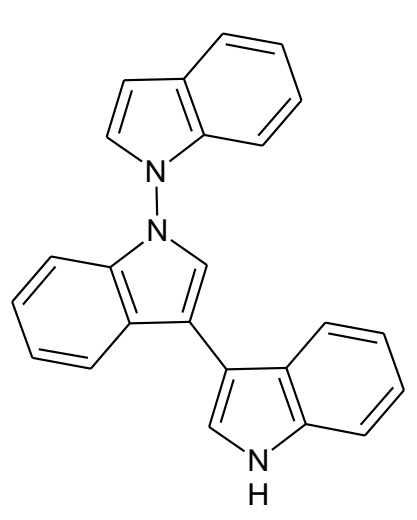

$1,1^{\prime}-3,3^{\prime}$

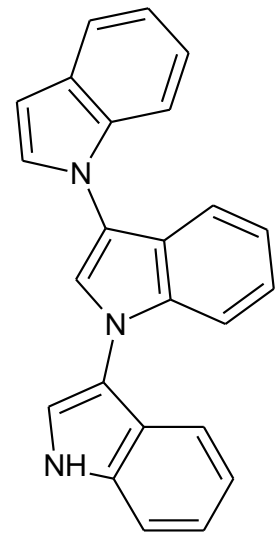

1,3

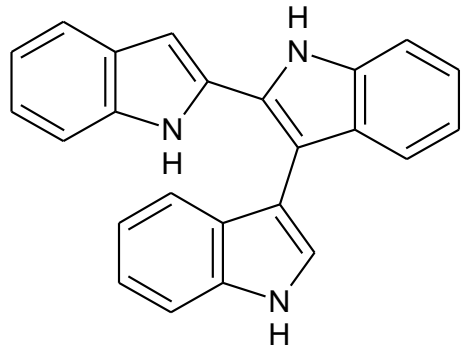

$2,2^{\prime}-3,3^{\prime}$

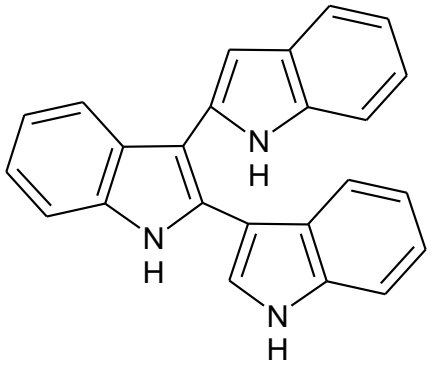

2,3

Most approaches reported to date for the polymerization of indole involve electrochemical oxidation or metal-salt catalyzed chemical synthesis. In all the cases the polymer produced is in the doped form and therefore does not exhibit significant photoluminescence. Moreover the use of harsh and hazardous conditions for the polymerization does not allow the direct utilization (without purification and separation of the dopant) of polyindole in biosensing applications. Enzymatic catalysis is an environmentally benign route for the synthesis of conjugated polymers in predominantly aqueous environment. Polypyrrole, polyaniline, and poly(3,4-dioxyethylene thiophene) (PEDOT) have been successfully synthesized with improved yield and greater reaction control by using enzymes, such as horseradish peroxidase (HRP) or soybean peroxidase (SBP), as catalysts [12-15]. The goal of this work was to explore the possibility of using an enzyme catalyst to create fluorescent indole species suitable for biosensor applications.

\section{Experimental Section}

\subsection{Chemical Reagents}

Indole (purity > 99\%), poly(styrene sulfonic acid)-sodium salt [PSS] (MW = 70,000 Daltons), anhydrous citric acid (purity 99\%) and sodium citrate monohydrate (purity 99\%), absolute ethanol, hydrogen peroxide (30\% wt) were purchased from Sigma-Aldrich Co., St. Louis, MO, USA. 3\% (w/v) stock solutions of $\mathrm{H}_{2} \mathrm{O}_{2}$ were prepared by diluting the $30 \%$ solution using DI water and stored in the refrigerator before and after use. 
Soybean Peroxidase (SBP) (EC 1.11.1.7) was purchased from Bioresearch Products Inc. North Liberty, IA and obtained as a reddish brown powder. SBP with an RZ (Reinheitszhal value) of 2.8 and a specific activity of 1840 IU per mg dry weight was used for all reported experiments. Prior to use SBP was dissolved in deionized water to a stock solution concentration of $3 \mathrm{mg} / \mathrm{mL}$. Specific activity was assessed through an established guaiacol oxidation assay [16].

DI water was obtained from a Millipore Elix 3 system equipped with Proguard 2 filters. The system produces DI water with a resistance greater than $15 \mathrm{Mohm} \cdot \mathrm{cm}$.

\subsection{Procedure}

An amount of $28 \mathrm{mg}$ of indole was dissolved in $3 \mathrm{~mL}$ of $30 \%(\mathrm{v} / \mathrm{v})$ ethanol in deionized water for a monomer concentration of $80 \mathrm{mM}$. If the reaction was run in a buffered solution, a $\mathrm{pH} 4.0,100 \mathrm{mM}$ citrate buffer was used instead of deionized water. If a surfactant or polyelectrolytes was used for the reaction, $150 \mathrm{mg}$ of Triton X-100 $(80 \mathrm{mM})$ or $55 \mathrm{mg}$ of PSS $(80 \mathrm{mM})$ was then added. To the indole solution, $300 \mu \mathrm{L}$ of enzyme (SBP) stock solution was then added. Immediately, after the addition of SBP, an initial aliquot of $22.6 \mu \mathrm{L}$ of $3 \% \mathrm{H}_{2} \mathrm{O}_{2}$ was added. Subsequent additions of equal volume and concentration were made at an interval of $5 \mathrm{~min}$ for one hour. The reaction was allowed to stir at room temperature $\left(\sim 21{ }^{\circ} \mathrm{C}\right)$ for an additional hour. The final product was isolated by diluting the reaction mixture with deionized water and dialyzing the resulting compound in a dialysis bag (Molecular weight cut-off 500 Dalton) against deionized water.

\subsection{Characterization}

The absorption spectra of the monomer and all polymeric products were obtained on an Agilent 8453 photodiode array UV-visible spectrometer in a $1 \mathrm{~cm}$ quartz cuvette. For absorption measurements, a $100 \mu \mathrm{L}$ aliquot of the reaction mixture was added to $3 \mathrm{~mL}$ solution of $30 \%$ ethanol in DI water. The fluorescence spectra of the monomer and polymeric products were obtained using Agilent Cary Eclipse Fluorescence Spectrophotometer in a $1 \mathrm{~cm}$ quartz cuvette. Samples were prepared by dissolving $30 \mu \mathrm{L}$ of the reaction solution in $3 \mathrm{~mL}$ of absolute ethanol. Dilute concentrations were purposely used to limit self-quenching due to aggregation. Fourier Transform Infrared (FTIR) Spectroscopy measurements were carried out on a Thermo Scientific Nicolet 4700 FTIR with a Smart Orbit Attenuated total reflectance (ATR) accessory. ${ }^{1} \mathrm{H}-\mathrm{NMR}$ measurements were recorded on a Bruker Avance DRX $500 \mathrm{MHz} \mathrm{NMR}$ in deuterated dimethyl sulfoxide (DMSO).

\section{Results and Discussion}

The oxidative bulk polymerization of indole reported in the literature is most commonly carried out in organic solvents and is catalyzed by strong oxidizing agents [17]. Electrochemical polymerization of indole in acetonitrile in the presence of electrolytes such as $\mathrm{LiClO}_{4}$ or $\mathrm{LiBF}_{4}$, has also been reported [18]. All of these methods of polymerization involve non-biocompatible organic solvents and/or metal catalysts. Moreover, significant purification is essential to obtain the polymer in the fluorescent form and render it useful for biosensing or imaging applications. 


\subsection{Enzyme Catalysis}

Peroxidases are known to catalyze the polymerization of certain types of aromatic amines, thiophenes and pyrroles in mildly acidic conditions ( $\mathrm{pH} 3.5-5)$ to form conjugated polymers $[12,19,20]$. In these reactions anionic polyelectrolytes such as PSS are used as templates to (1) improve solubility of the monomer and the polymer (2) electrostatically interact with the radical cationic species of the oxidized monomer and the growing polymer chain, and (3) serve as a charge balancing dopant for the polymer. While the template facilitates the reaction, the products formed are often doped and non-fluorescent.

\subsection{Enzymatic Polymerization of Indole}

In an attempt to develop a mild and possibly biocompatible method, here we extend the use of peroxidases for the polymerization of undoped and fluorescent indoles.

Our initial investigations on the enzymatic polymerization of indole indicated that electrically conducting polymers of indole can be synthesized in mildly-acidic buffered solutions in the presence of an anionic dopant/template, such as PSS [21]. However, biosensors that are based on measurement of electronic properties of conjugated electroactive polymers have already been reported. However, from these initial reactions and through the use of FTIR spectroscopy, we observed that polyindoles synthesized enzymatically exhibit a characteristic absorption of the $\mathrm{N}-\mathrm{H}$ bond at $\sim 3398 \mathrm{~cm}^{-1}$. This band together with the band at $1570 \mathrm{~cm}^{-1}$ can be ascribed to be the stretching and deformation vibrations of the N-H bond, respectively [22]. The full spectrum is provided in the Appendix as Figure A1. This supports the argument corroborated by other independent studies that the $2-3$ coupling (Figure 2 ) is the most probable route among the four possible coupling mechanisms shown in Figure 1 [23].

Figure 2. Reaction scheme and proposed coupling route for the biocatalytic polymerization of indole with SBP.
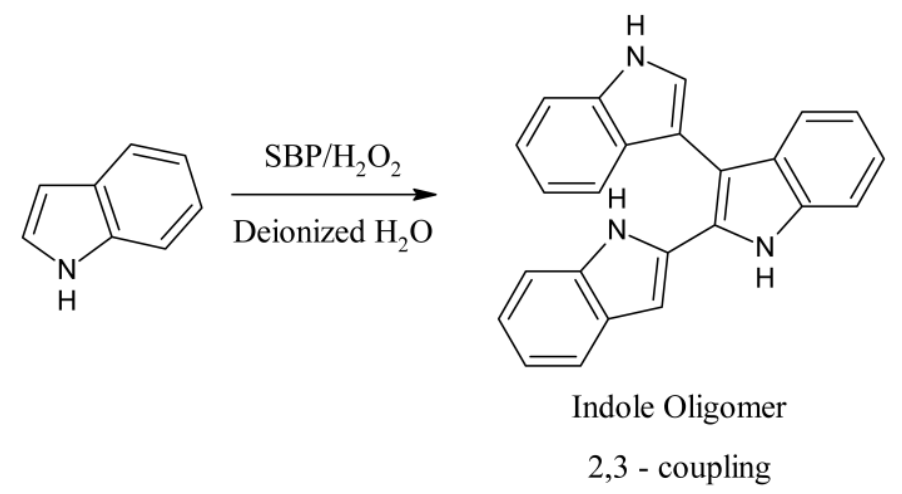

During the characterization of the polyindole-PSS reaction mixture, a faint green fluorescence emission was observed. Upon further purification by precipitating the polyindole-PSS complex, the emission was determined to be from soluble co-products in the reaction mixture and not from the PSS complexed polyindole. This is also supported by our earlier work that has showed that the enzyme-catalyzed polymerization of pyrrole progressed more efficiently to yield an electrically conducting product in the presence of buffered solutions and PSS [18]. However, the generation of an 
electrically conducting species typically leads a quenched fluorescence emission. Based on previous reports, this luminescence was most likely from soluble undoped oligomeric products [24]. Given this prior work and our recent work on pyrrole oligomer sensors [25], we designed a series of experiments to create fluorescent indoles for biosensor applications. To validate the role of SBP and hydrogen peroxide in the oligomerization reaction, control experiments were performed without SBP but with hydrogen peroxide in the reaction mixture. Similarly another reaction was performed with SBP alone but without the addition of hydrogen peroxide. The UV-Vis spectra from these experiments, provided in the Appendix as Figure A2, clearly indicate that the enzyme catalyzes the oligomerization and the reaction does not proceed in the absence of either SBP or hydrogen peroxide.

In an effort to observe the evolution of the fluorescent conjugated species of indole a series of experiments were carried out. Buffers salts and charged templates are known to interact with or dope growing conjugated polymer chains through electrostatic interactions with radical cations along the polymer backbone. To confirm these deleterious effects some initial experiments were performed on the polymerization of indole catalyzed by SBP in a buffered solution, a buffered solution with PSS, and as a control in deionized water without a buffer or PSS. Additionally, all of the reactions contained ethanol as a cosolvent to help solubilize the indole monomer. Using ethanol as a cosolvent has previously been reported to have little effect on the activity of SBP [26]. In the presence of $30 \%$ ethanol, SBP maintains roughly $80 \%$ of its original activity, making it a viable catalyst for these investigations [27]. When observed under UV light $(365 \mathrm{~nm})$, it is clear that a strongly fluorescent species is formed in the absence of a buffer and PSS, Figure 3. Within the first minute, the reaction containing DI water emits a strong blue/green fluorescence. Reactions containing buffer or buffer and PSS exhibit an emission that is either dramatically diminished or not present. A less intense, but still prevalent emission was also observed when the reaction was run in pure DI water without an ethanol cosolvent.

Figure 3. Image of the initial stages of the enzymatic polymerization of indole under $365 \mathrm{~nm}$ blacklight in the presence of DI water, citrate buffer, and buffer and PSS. All solutions contain $30 \%$ ethanol as cosolvent.

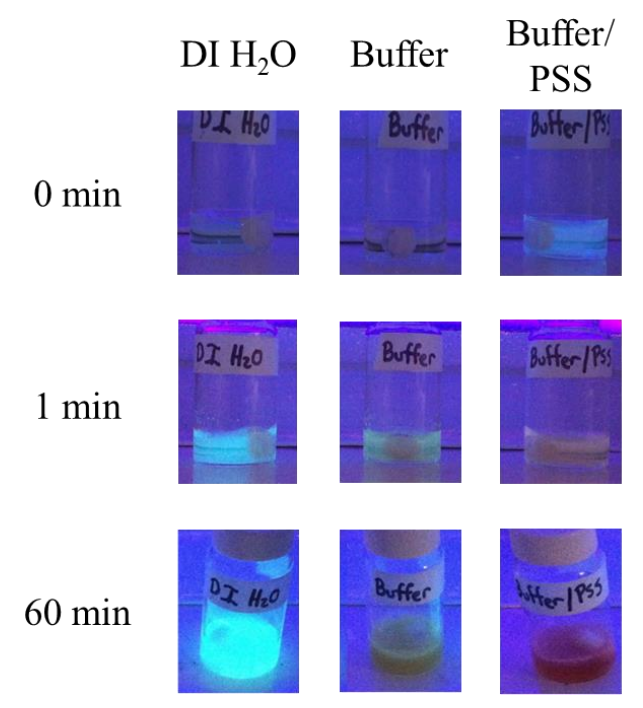

Upon isolation of the product synthesized in DI water by dialysis, further structural characterization was performed. ${ }^{1} \mathrm{H}-\mathrm{NMR}$ analysis in deuterated DMSO revealed the final product is likely an oligomer 
of indole coupled between the 2 and 3 positions. No evidence of any ring opened products was observed as previously reported for the oxidation of indole by horseradish peroxidase [24]. The ${ }^{1} \mathrm{H}-\mathrm{NMR}$ spectrum is provided in the Appendix as Figure A3.

\subsection{Reaction Monitoring with Absorption and Fluorescence Spectroscopy}

To further investigate the emergence of fluorescence during the enzymatic polymerization, absorption and fluorescence spectra of the reaction were monitored over the course of one hour. Figure 4a presents the absorption spectra of the reaction in a DI water/ethanol mixture, as well as in the presence of Triton X-100, a nonionic surfactant. Triton X-100 was selected to improve the solubility of the growing polymer chain without doping the backbone. The activity of SBP is not drastically influenced by the presence of Triton X-100. As reported earlier, SBP is active in the presence of Triton $\mathrm{X}-100$ and has been used for treatment of phenol in wastewater [28].

The initial reaction solution containing only solvent, monomer, and enzyme was colorless. Upon the addition of SBP and the first aliquot of $\mathrm{H}_{2} \mathrm{O}_{2}$, the reaction solution turns a pale orange. The reaction continues to deepen in color with additional aliquots of hydrogen peroxide. After an hour, a small amount of precipitate is observed in the reaction solution. A similar progression is observed for the reaction containing Triton $\mathrm{X}-100$, however the reaction solution always remained less colored compared to reactions without surfactant. For comparison, the post-reaction absorption spectra for the polymerization in the presence PSS and buffered solutions are included as Figure 4b.

Figure 4. (a) Absorption spectra development during unbuffered polymerization reaction.

(b) Comparison of absorption spectra of indole monomer and final products synthesized in a buffer in the presence of PSS and Triton X-100.
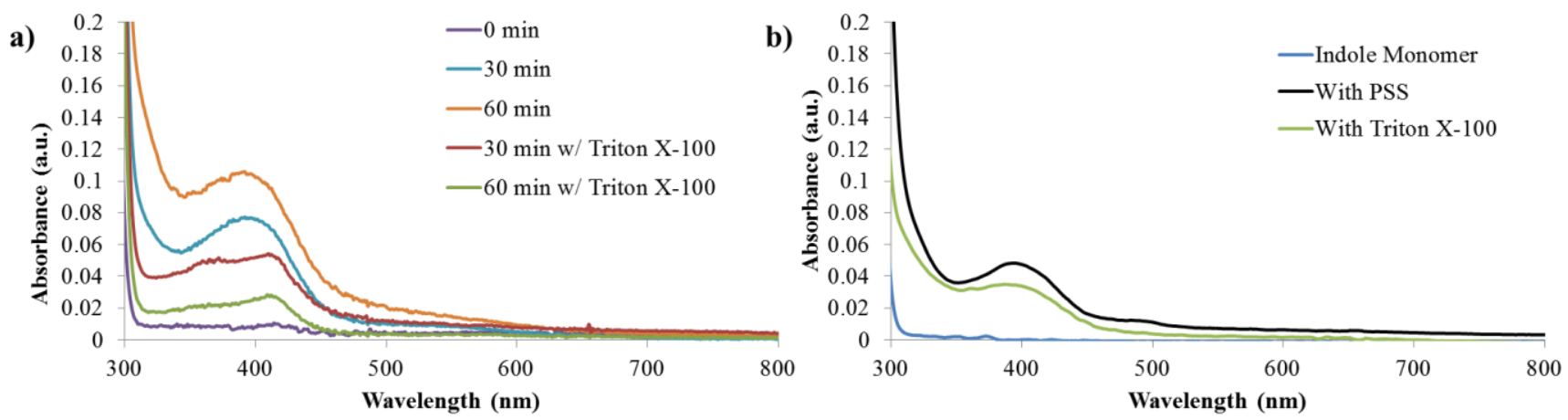

For all reactions, absorption from the indole monomer is observed at $300 \mathrm{~nm}$, Appendix Figure A4. As the reactions proceed, new peaks are generated at $390 \mathrm{~nm}$ and $410 \mathrm{~nm}$ for solutions without and with Triton X-100, respectively. In both cases, the newly formed peak near $400 \mathrm{~nm}$ is believed to be due to the absorption of an indole species with extended conjugation. Similar spectra have previously been reported for polyindoles complexed with DNA when synthesized using the chemical oxidant ferric chloride [29]. As expected, the absorption peak for the reaction products in DI Water/ethanol increases over time as the concentration of absorbing species increases. In contrast, in the presence of Triton X-100, the absorption peak initially increases, but decreases significantly between 30 min and $60 \mathrm{~min}$. It is unclear why this occurs, however, it could be due to the formation of a less soluble species. 
Additionally based on the strength of the absorption peak, the concentration of absorbing species in solution is always higher for the reaction run without Triton $\mathrm{X}-100$. This could be the result of a higher concentration of indole monomer interacting with the enzyme when Triton X-100 is not present in the reaction. The enzyme is likely to remain within the bulk phase of the solution and not inside the surfactant molecules. Therefore, in reactions containing Triton X-100, only indole that is not partitioned inside the micelle is free to react with the enzyme. This could ultimately leads to a lower concentration of products formed.

Fluorescence emission $\left(\lambda_{\mathrm{ex}}=350 \mathrm{~nm}\right)$ during the polymerization reactions with and without Triton X-100 increases steadily over the course of one hour, Figure 5. This is expected as the number of emitters generated by the polymerization reaction increases over time. The emission maximum for both reactions is approximately $465 \mathrm{~nm}$ and does not shift upon excitation at higher wavelengths. The fluorescence emissions observed here are similar to those previous reported [26,30]. The reaction run without a surfactant/template has emission intensity almost five times greater than the reaction with Triton X-100. This corresponds well to the absorption data that suggest there is a higher concentration of absorbing/emitting products in solution for the reaction without a surfactant. Emission spectra monitoring at excitation wavelengths of 300 (monomer excitation) and $420 \mathrm{~nm}$ are included as Figures A5 and A6 in the Appendix.

Figure 5. Fluorescence emission $\left(\lambda_{\mathrm{ex}}=350 \mathrm{~nm}\right)$ as a function of reaction time.
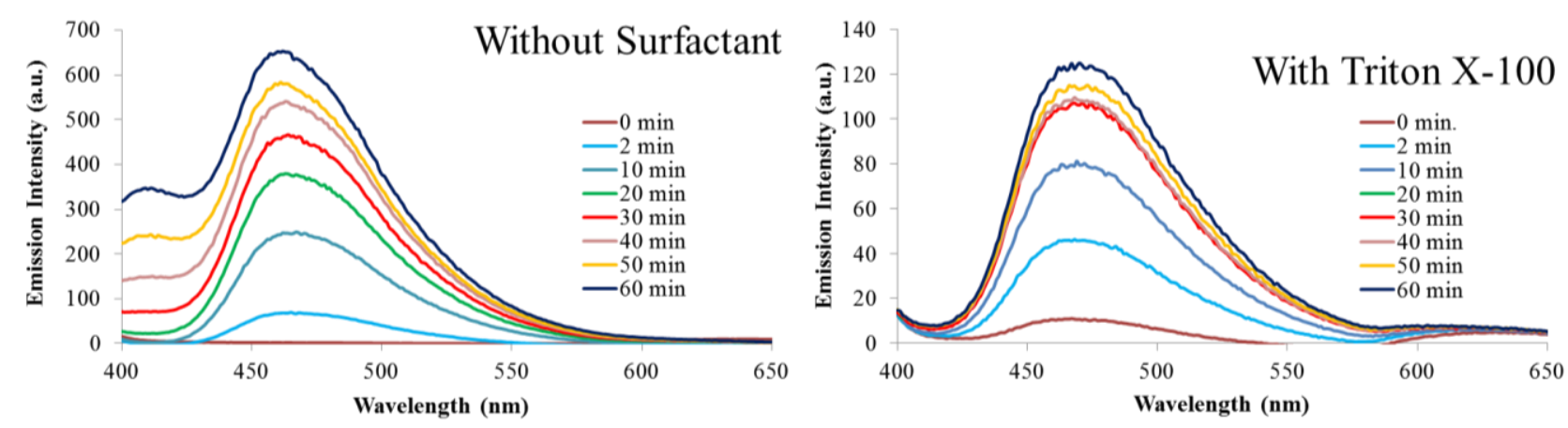

Figure 6. Rate of Normalized Emission Growth at $465 \mathrm{~nm}$ during Polymerization $\left(\lambda_{\mathrm{ex}}=350 \mathrm{~nm}\right)$.

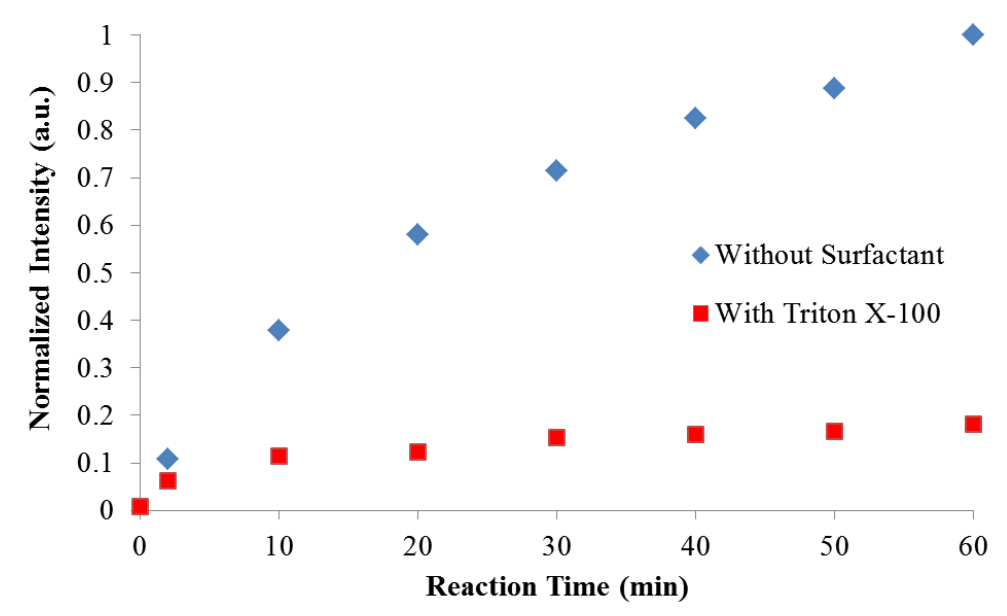

Figure 6 presents a comparison of the rate of normalized emission intensity growth. The spectra were normalized to the emission intensity present $60 \mathrm{~min}$ after the start of the reaction without a 
surfactant. In both reactions there is a rapid increase in fluorescence emission within the first two minutes. This is due to an extended conjugated length created from the initial coupling of indole monomers along the growing chain. When Triton X-100 is present, the rate of emission growth begins to level off after $30 \mathrm{~min}$; when no surfactant was present the emission intensity continues to increase steadily over the course of the first hour. Fluorescence monitoring was stopped at one hour when a precipitate was observed in the reaction without Triton X-100.

\section{Conclusions}

In conclusion, an improved biocatalytic route is presented here that allows for the easy creation of fluorescent conjugated materials under near neutral $\mathrm{pH}$ conditions in biocompatible solvents such as water and ethanol. The enzyme-catalyzed technique is simple and offers a direct route to a conjugated indole oligomer with photoluminescent properties without exhaustive purification steps. The polymerization is sensitive to the presence of anionic buffers and templates that may dope the newly formed conjugated chain and quench the photoluminescence. In situ monitoring of absorption and emission spectra shows that when the reaction is conducted in just deionized water with an ethanol cosolvent, a more photoluminescent material is created. The presence of the non-ionic surfactant, Triton X-100 during the reaction results in decreased photoluminescence intensity. ${ }^{1} \mathrm{H}-\mathrm{NMR}$ and FTIR analysis suggests that the biocatalytically coupled indoles are linked at the 2 and 3 positions on the indole ring. This improved synthesis technique offers the possibility for enhancing the interactions of fluorescent conjugated materials with biomolecules, rendering them suitable for biosensing and imaging applications.

\section{Acknowledgments}

The authors thank Zarif Farhana Mohd Aris and Wendy Gavin for their help obtaining ${ }^{1} \mathrm{H}-\mathrm{NMR}$ spectra. The authors also thank Prof. Daniel Sandman for his suggestions and useful discussions.

\section{Author Contributions}

Ryan M. Bouldin and Ankita Singh conducted laboratory synthesis and characterization of the polyindole as well as writing significant portions of the manuscript. These two authors contributed equally to the paper. Michael Magaletta and Sean Connor assisted in conducting experiments for the paper in the laboratory. Jayant Kumar and Ramaswamy Nagarajan supervised, reviewed the work and helped in the design of the laboratory experiments and the interpretation of the results.

\section{Conflicts of Interest}

The authors declare no conflict of interest.

\section{References}

1. Guiseppi-Elie, A.; Wallace, G.G.; Matsue, T. Chemical and Biological Sensors Based on Electrically Conducting Polymers. In Handbook of Conducting Polymers, 2nd ed.; Skotheim, T.A., Elsenbaumer, R.L., Reynolds, J.R., Eds.; Marcel Dekker Inc.: New York, NY, USA, 1997; pp. 963-988. 
2. Feng, X.; Liu, L.; Wang, S.; Zhu, D. Water-soluble fluorescent conjugated polymers and their interactions with biomacromolecules for sensitive biosensors. Chem. Soc. Rev. 2010, 39, 2411-2419.

3. Liu, B.; Bazan, G.C. Homogeneous fluorescence-based DNA detection with water-soluble conjugated polymers. Chem. Mater. 2004, 16, 4467-4476.

4. Chan, Y.-H.; Wu, P.-J. Semiconducting polymer nanoparticles as fluorescent probes for biological imaging and sensing. Part. Part. Syst. Char. 2014, doi:10.1002/ppsc.201400123.

5. Faïd, K.; Leclerc, M. Responsive supramolecular polythiophene assemblies. J. Am. Chem. Soc. 1998, 120, 5274-5278.

6. Hussain, M.; Wackerlig, J.; Lieberzeit, P.A. Biomimetic strategies for sensing biological species. Biosensors 2013, 3, 89-107.

7. Chen, L.; McBrach, G.C.; Wang, H.-L.; Helgeson, R.; Wudl, R.; Whitten, D.G. Highly sensitive biological and chemical sensors based on reversible fluorescence quenching in a conjugated polymer. Proc. Natl. Acad. Sci. USA 1996, 96, 12287-12292.

8. Bridges, J.W.; Williams, R.T. The fluorescence of indoles and aniline derivatives. Biochem. J. 1968, 107, 225-237.

9. John, A.; Palaniappan, S. Synthesis and characterization of soluble poly(N-heptyl indole). Polymer 2005, 46, 12037-12039.

10. Zotti, G.; Zecchin, S.; Schiavon, G.; Seraglia, R.; Berlin, A.; Canavesi, A. Structure of polyindoles from anodic coupling of indoles: an electrochemical approach. Chem. Mater. 1994, 6, 1742-1748.

11. Cai, Z.; Zhang, R.; Shi, X. Preparation and characterization of polyindole nanofibers by electrospinning method. Synthetic Met. 2012, 162, 2069-2074.

12. Liu, W.; Kumar, J.; Tripathy, S.; Senecal, K.J.; Samuelson, L. Enzymatically synthesized conducting polyaniline. J. Am. Chem. Soc. 1999, 1, 71-78.

13. Liu, W.; Cholli, A.L.; Nagarajan, R.; Kumar, J.; Tripathy, S.; Bruno, F.F.; Samuelson, L. The role of template in the enzymatic synthesis of conducting polyaniline. J. Am. Chem. Soc. 1999, 121, $11345-11355$.

14. Bruno, F.F.; Fossey, S.A.; Nagarajan, S.; Nagarajan, R.; Kumar, J.; Samuelson, L.A. Biomimetic synthesis of water-soluble conducting copolymers/homopolymers of pyrrole and 3,4-ethylenedioxythiophene. Biomacromolecules 2006, 7, 586-589.

15. Ravichandran, S; Nagarajan, S.; Kokil, A.; Ponrathnam, T.; Bouldin, R.M.; Bruno, F.F.; Samuelson, L.; Kumar, J.; Nagarajan, R. Micellar nanoreactors for hematin catalyzed synthesis of electrically conducting polypyrrole. Langmuir 2012, 28, 13380-13386.

16. Sessa, D.J.; Anderson, R.L. Soybean peroxidases: Purification and some properties. J. Agric. Food Chem. 1981, 29, 960-965.

17. Billaud, D.; Maarouf, E.B.; Hannecart, E. Chemical oxidation and polymerization of indole. Synthetic Met. 1995, 69, 571-572.

18. Billaud, D.; Maarouf, E.B.; Hannecart, E. Electrochemical polymerization of indole. Polymer 1994, 35, 2010-2011.

19. Tewari, A.I.; Kokil, A.; Ravichandran, S.; Nagarajan, S.; Bouldin, R.; Bruno, F.F.; Samuelson, L.A.; Nagarajan, R.; Kumar, J. Soybean peroxidase catalyzed enzymatic synthesis of pyrrole/EDOT copolymers. Macromol. Chem. Phys. 2010, 211, 1610-1617.

20. Bouldin, R.; Ravichandran, S.; Kokil, A.; Garhwal, R.; Nagarajan, S.; Kumar, J.; Bruno, F.F.; Samuelson, L.A.; Nagarajan, R. Synthesis of polypyrrole with fewer structural defects using enzyme catalysis. Synthetic Met. 2011, 161, 1611-1617. 
21. Nagarajan, S.; Jayaraman, S.; Ravichandran, S.; Bouldin, R.; Nagarajan, R.; Kumar, J. Enzyme Mediated Synthesis as an Eco-Friendly Tool for Chemical Education. In Proceedings of the American Chemical Society National Meetings, San Diego, CA, USA, 25-29 March 2012.

22. An, S.; Abdiryim, T.; Ding, Y.; Nurulla, I. A comparative study of the microemulsion and interfacial polymerization for polyindole. Mater. Lett. 2008, 62, 935-938.

23. Talbi, H.; Ghanbaja, J.; Billaud, D.; Humbert, B. Vibrational properties and structural studies of doped and dedoped polyindole by FTIR, Raman and EEL spectroscopies. Polymer 1997, 38, 2099-2106.

24. Ximenes, V.F.; Campa, A.; Catalani, L.H. The oxidation of indole derivatives catalyzed by horseradish peroxidase is highly chemiluminescent. Arch Biochem Biophys. 2001, 387, 173-179.

25. Bouldin, R.M.; Kokil, A.; Ponrathnam, T.; Urban, N.; Kumar, J.; Samuelson, L.A.; Nagarajan, R. Biocatalyic synthesis of unusually photoluminescent oligomers and electrically conducting polymers of 4-(3-pyrrolyl)butyric acid. J. Appl. Poly. Sci. 2014, 131, doi:10.1002/app.41035.

26. Kim, Y.H.; An, E.S.; Song, B.K.; Kim, D.S.; Chelikani, R. Polymerization of cardanol using soybean peroxidase and its potential application as anti-biofilm coating material. Biotechnol. Lett. 2003, 25, 1521-1524.

27. Ghaemmaghami, F.I.; Alemzadeh, I.; Motamed, S. Seed coating soybean peroxidase: Extraction and biocatalytic properties determination. Iranian J. Chem. Eng. 2010, 7, 28-38.

28. Steevensz, A.; Madur, S.; Feng, W.; Taylor, K.E.; Bewtra, J.K.; Biswas, N. Crude soybean hull peroxidase treatment of phenol in synthetic and real wastewater: Enzyme economy enhanced by Triton X-100. Enzym. Microb. Tech. 2014, 55, 65-71.

29. Hassanien, R.; Al-Hinai, M.; Al-Said, S.A.F.; Little, R.; Siller, L.; Wright, N.G.; Houlton, A.; Horrocks, B.R. Preparation and characterization of conductive and photoluminescent DNA-templated polyindole nanowires. ACS Nano 2010, 4, 2149-2159.

30. Xu, J.; Nie, G.; Zhang, S.; Han, X.; Hou, J.; Pu, S. Electrosynthesis of free-standing polyindole films in borontrifluoride diethyl etherate. J. Polymer Sci. Polymer Chem. 2005, 43, 1444-1453.

\section{Appendix}

\section{FTIR of Indole Monomer and Enzyme Catalyzed Polyindole}

Figure A1. FTIR-ATR spectra of indole and polyindole.

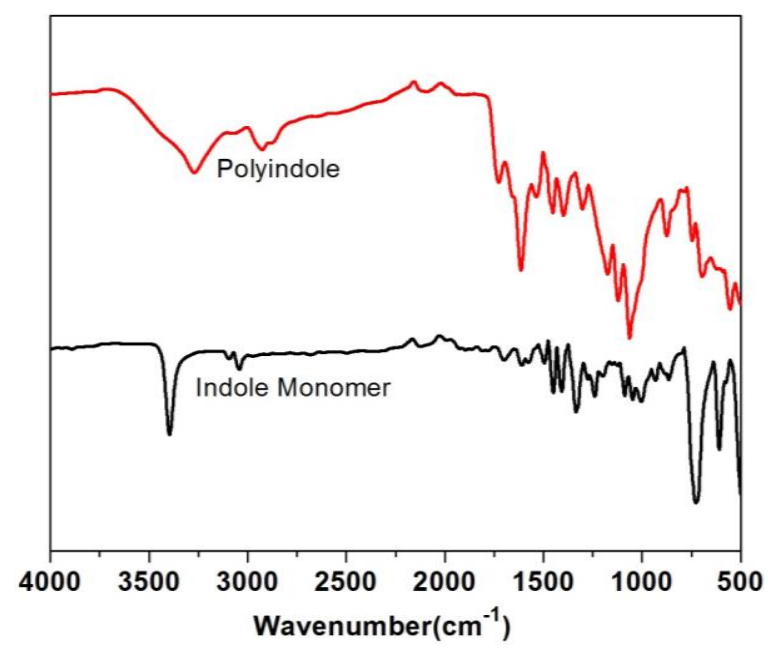




\section{Absorbance Spectra Monitoring}

Figure A2. Absorbance spectra monitoring of control reactions with and without SBP and hydrogen peroxide. Measurement recorded from $10 \mu \mathrm{L}$ reaction sample in $290 \mu \mathrm{L}$ of solvent system.

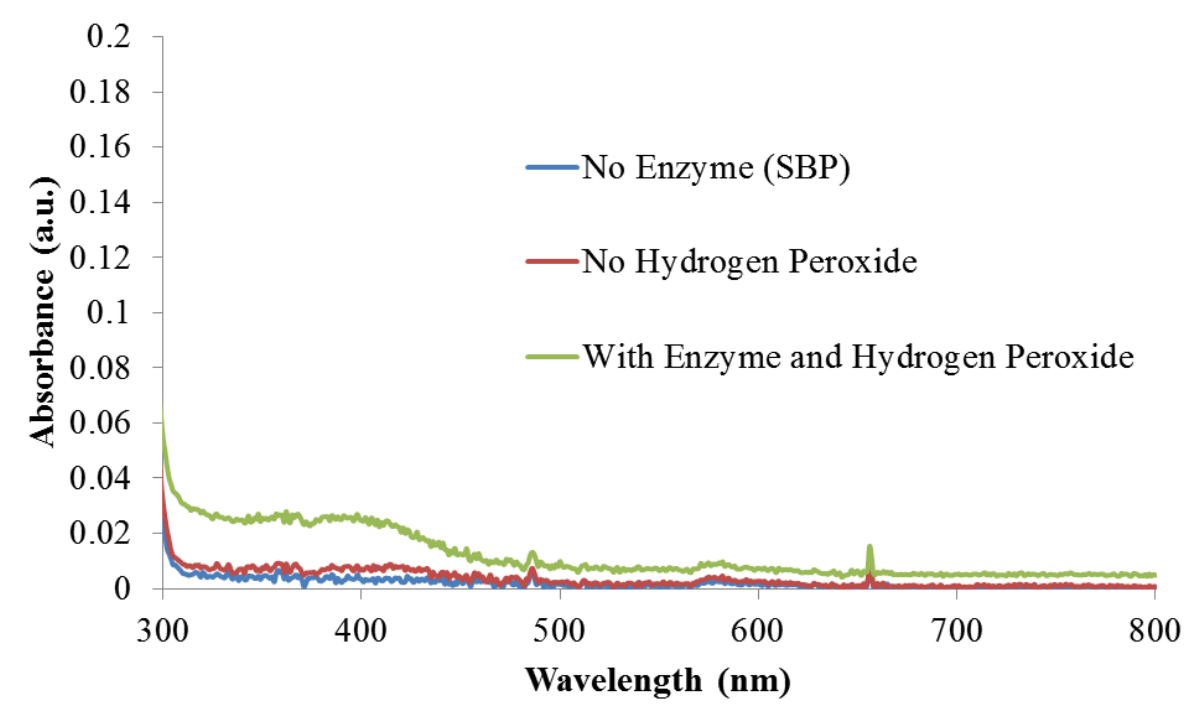

\section{${ }^{1}$ H-NMR Analysis of Indole Oligomer in d-DMSO}

Figure A3. ${ }^{1} \mathrm{H}-\mathrm{NMR}$ of SBP catalyzed polymerization product of indole in deionized water and ethanol.

Polyindole 09162014/1

H-NMR

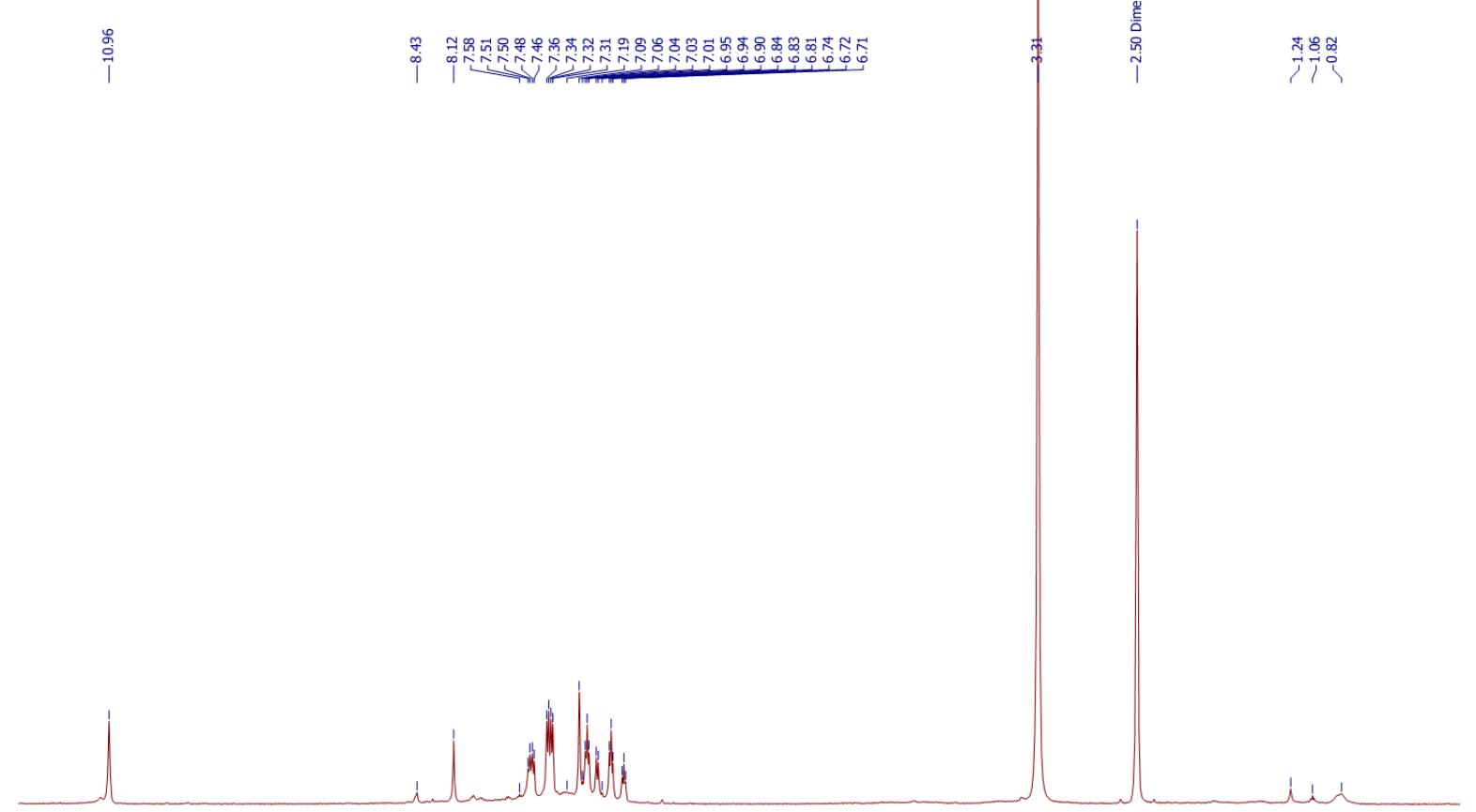

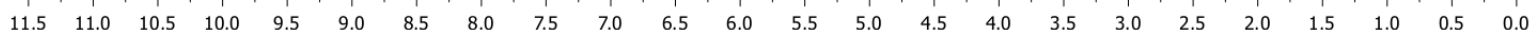




\section{Absorbance Spectra Monitoring}

Figure A4. Full absorbance spectra monitoring of reaction with and without Triton X-100.

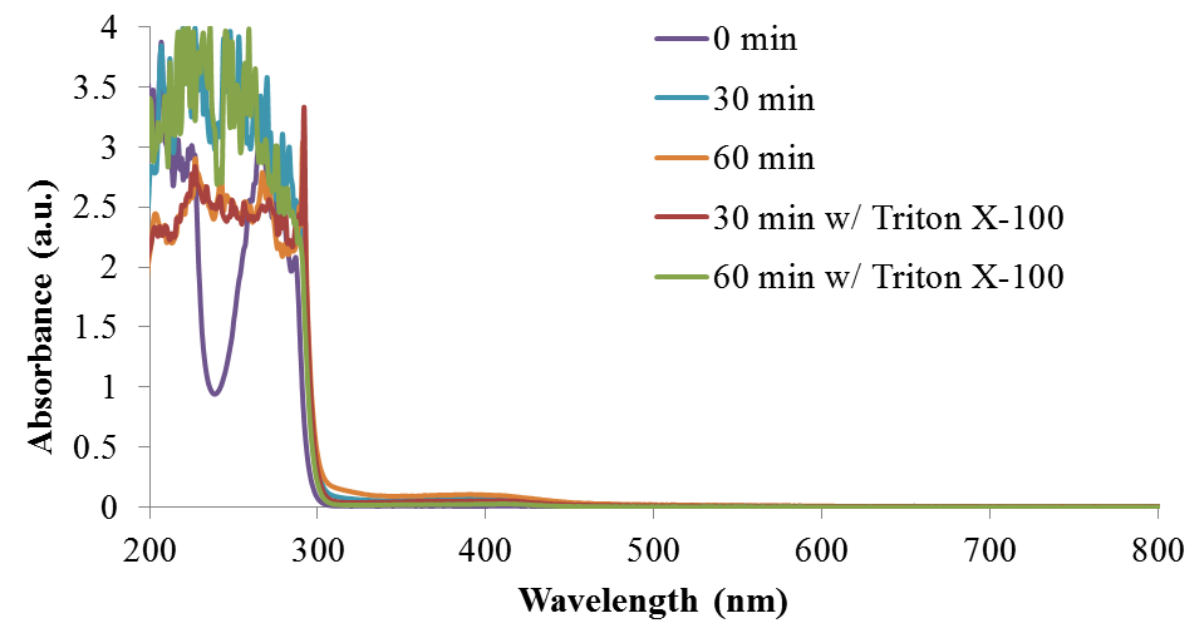

\section{Photoluminescence Monitoring at Various Excitation Wavelengths}

Figure A5. Fluorescence emission $\left(\lambda_{\mathrm{ex}}=300 \mathrm{~nm}\right)$ as a function of reaction time with and without Triton X-100. Excitation at $300 \mathrm{~nm}$ corresponds to excitation of the indole monomer.

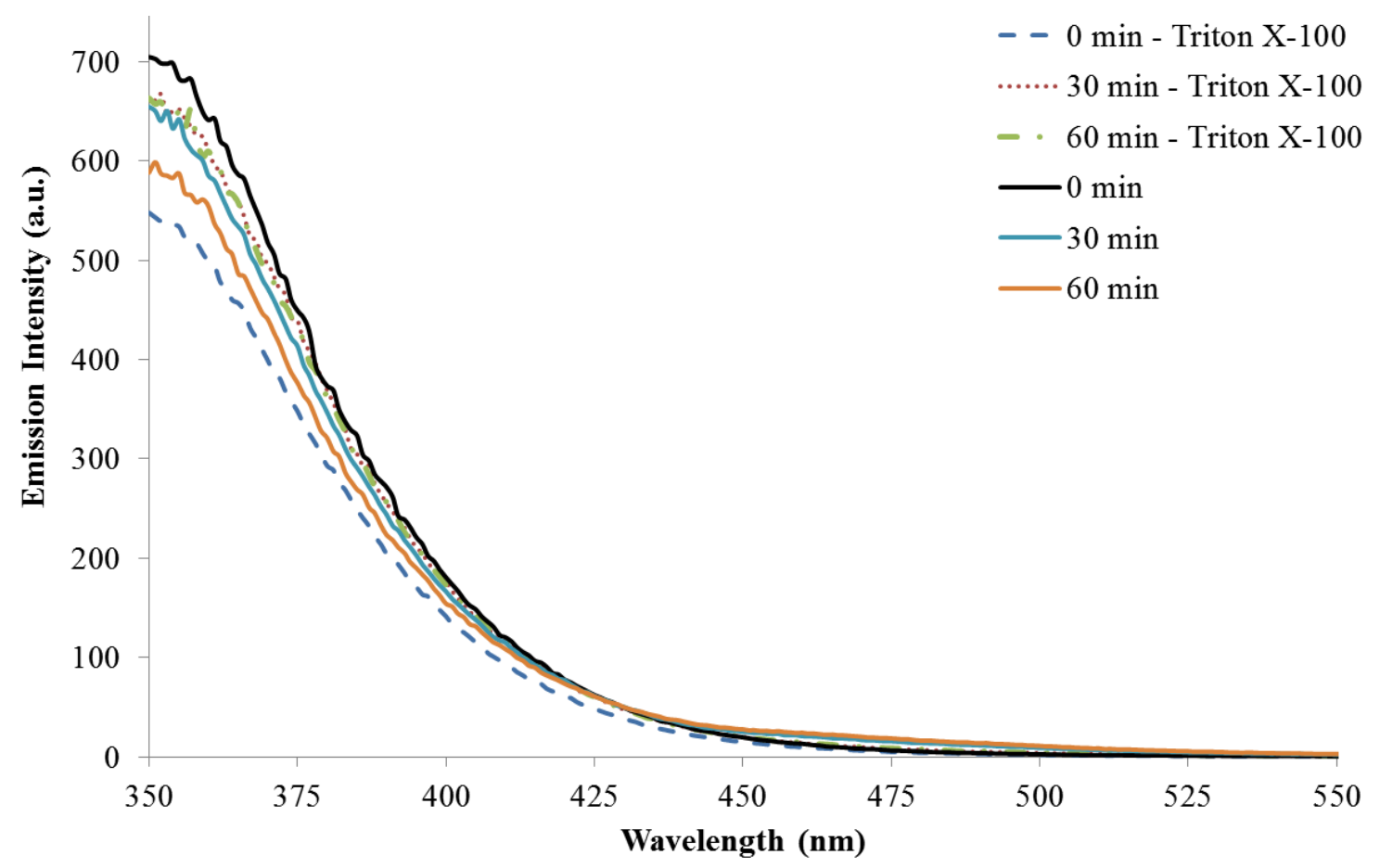


Figure A6. Fluorescence emission $\left(\lambda_{\mathrm{ex}}=420 \mathrm{~nm}\right)$ as a function of reaction time with and without Triton X-100.

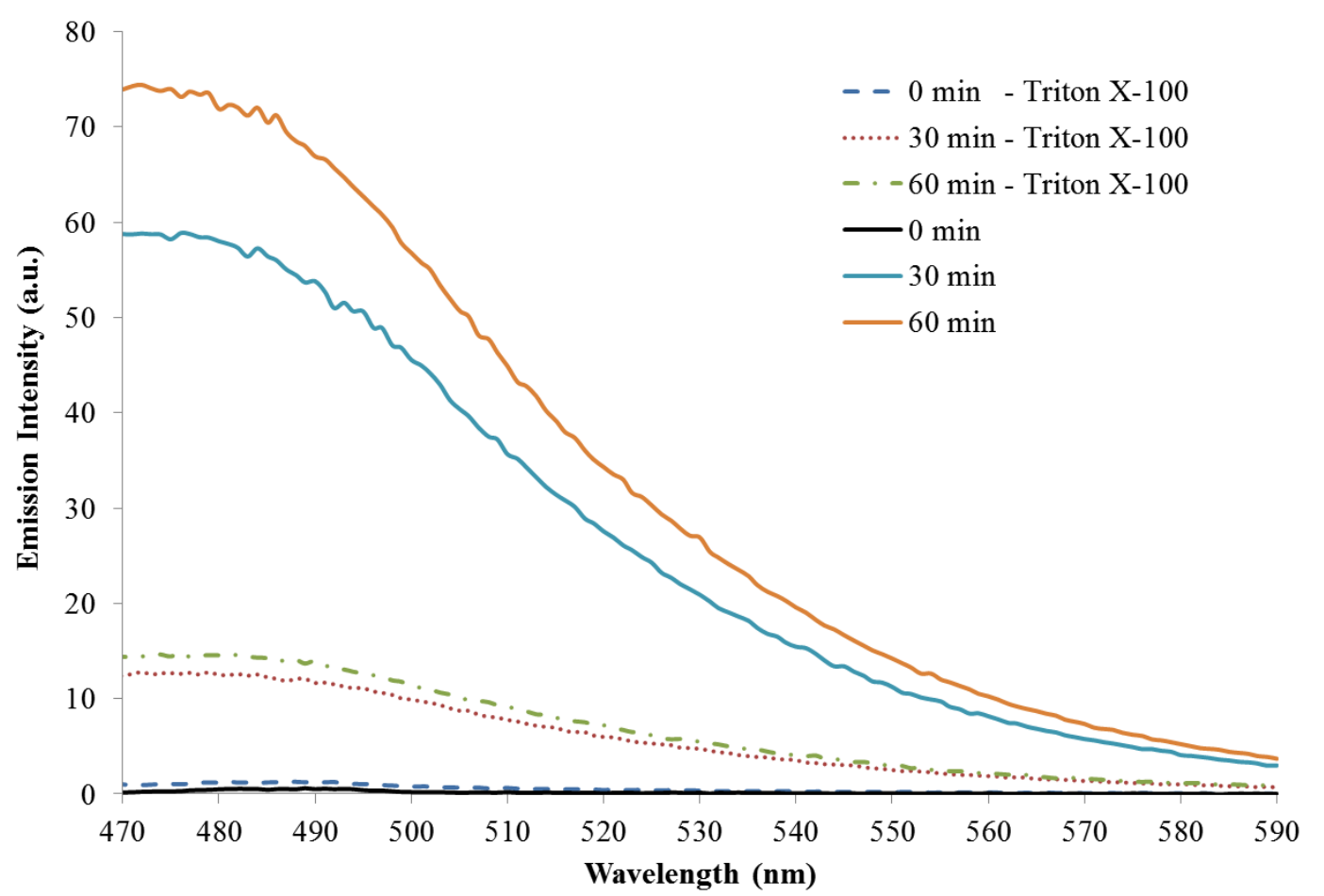

(C) 2014 by the authors; licensee MDPI, Basel, Switzerland. This article is an open access article distributed under the terms and conditions of the Creative Commons Attribution license (http://creativecommons.org/licenses/by/4.0/). 